\title{
Ultrasonic Intra-body Networking: Interference Modeling, Stochastic Channel Access and Rate Control
}

\author{
Zhangyu Guan $^{\dagger \ddagger}$, G. Enrico Santagati ${ }^{\dagger}$ and Tommaso Melodia ${ }^{\dagger}$ \\ ${ }^{\dagger}$ Department of Electrical and Computer Engineering, Northeastern University, Boston, MA

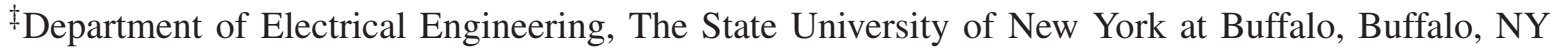 \\ Email: zguan2@buffalo.edu, \{santagati, melodia\}@ece.neu.edu
}

\begin{abstract}
We consider the problem of designing optimal network control algorithms for distributed networked systems of implantable medical devices wirelessly interconnected by means of ultrasonic waves, which are known to propagate better than radio-frequency electromagnetic waves in aqueous media such as human tissues. Specifically, we propose lightweight, asynchronous, and distributed algorithms for joint rate control and stochastic channel access designed to maximize the throughput of ultrasonic intra-body area networks under energy constraints.

We first develop (and validate through testbed experiments) a statistical model of the ultrasonic channel and of the spatial and temporal variability of ultrasonic interference. Compared to in-air radio frequency (RF), human tissues show a much lower propagation speed, which further causes unaligned interference at the receiver. It is therefore inefficient to perform adaptation based on instantaneous channel state information (CSI). Based on this model, we formulate the problem of maximizing the network throughput by jointly controlling the transmission rate and the channel access probability over a finite time horizon based only on a statistical characterization of interference. We then propose a fully distributed solution algorithm, and through both simulation and testbed results, we show that the algorithm achieves considerable throughput gains compared with traditional algorithms.
\end{abstract}

\section{INTRODUCTION}

Wireless intra-body networks of implantable biomedical devices have the potential to enable revolutionary clinical applications. Most existing research and commercial efforts to date have focused on communications along the body surface among devices that use traditional electromagnetic radio-frequency $(\mathrm{RF})$ carrier waves, leaving the root challenge of enabling networked intra-body miniaturized sensors and actuators that communicate through body tissues substantially unaddressed.

Commercial wireless medical implants [1], [2] that communicate through RF point-to-point links have recently become available, along with RF-based transceiver chips for medical implants [3]. However, these technologies tend to almost-blindly scale down traditional wireless technologies (e.g., Bluetooth, Zigbee) to the intra-body environment, with little or no attention to the peculiar characteristics and safety requirements of the human body. The human body is however

This material is based upon work supported in part by the National Science Foundation under grant CAREER CNS-1253309. composed (up to 65\%) of water, a medium through which $\mathrm{RF}$ waves do not propagate well. In addition, the medical community is still divided on the risks caused by exposure of human tissues to RF radiation - the World Health Organization classifies RF waves as "possibly carcinogenic to humans". Not less importantly, RF-based technologies are prone to malicious jamming attacks or to environmental interference from pervasively deployed existing RF communication systems that can undermine the reliability and security of the intra-body network, and ultimately the safety of the patient.

For these reasons, in [4]-[6] we proposed a different approach and explored the use of ultrasonic waves to wirelessly internetwork in-body devices, i.e., ultrasonic intra-body area networks. Acoustic waves, typically generated through piezoelectric materials, are known to propagate better than RF in dielectric media composed primarily of water. Since World War II, piezoelectrically generated acoustic waves have found application, among others, in underwater communications (typically at frequencies between 0 and $100 \mathrm{kHz}$ ), in indoor localization [7], and, massively, in ultrasonic medical imaging [8]. While communication at low frequencies requires sizable transducers, innovations in piezoelectric materials and fabrication methods, primarily driven by the need for resolution in medical imaging, have made miniaturized transducers at the micro and even nano scales [9] a reality; with examples of devices that have even reached clinical stages [10]. Moreover, the medical experience of the last decades has demonstrated that ultrasounds are fundamentally safe, as long as acoustic power dissipation in tissues is limited to predefined safety levels [4], [11].

Envisioned New Applications. Ultrasonic wave heat dissipation in tissues is low compared to RF waves [12]. Therefore, ultrasonic intra-body area networks can improve existing biomedical applications as well as enable a rich set of new applications, which can be as diverse as automated drug administration, pervasive surveillance using pill-size ingestible cameras, bio-hybrid implants, intraocular pressure monitoring, malicious agent monitoring, heart monitoring, and minimallyinvasive microsurgery [4].

While in some applications, e.g., in under-skin and static scenarios, implanted sensors can be easily pre-deployed, 
possibly wired, and scheduled in a centralized fashion, in many envisioned applications this is however undesirable. For example, in automated drug administration, targeted drug delivery in a spatial-, temporal- and dosage-controlled fashion based on distributed measures of biological parameters is already a reality [10]. In these scenarios, it is infeasible to wire deeply-implanted devices, and centralized scheduling of interfering wireless links serving different areas of the body may be complex and involve long-range, high-power transmissions that are undesirable due to potential excessive radiation. Another example is pervasive surveillance, where malicious agents are monitored through distributed networks of ingestible sensors [13] - in this case centralized control is by no means easy to implement without global network topology information; moreover it may not be easy to maintain global synchronization, which makes distributed control an appealing approach as in traditional mobile ad hoc networks.

Based on these premises, in this paper we design lightweight (i.e., based on local decisions taken through polynomialtime algorithms and with minimal message exchange), asynchronous (updates at different nodes are unsynchronized, i.e., as in [14]), and distributed (i.e., without centralized control) resource allocation algorithms. Our objective is to jointly control cross-layer networking functionalities of devices in an ultrasonic network (e.g., channel access, spectrum management, queuing and rate control) to optimize the network throughput with a constrained energy budget and while keeping the radiated power within safety limits.

\section{Related WORK AND PAPER CONTRIBUtions}

There is a large body of work on cross-layer optimization algorithms for wireless networks (see, among many others [15], [16]). However, algorithms proposed for RF wireless communications (i) do not consider the spatially and temporally uncertain ultrasonic environment; plus, (ii) they typically require coordination and instantaneous and synchronized control message exchanges that are not desirable in resourceconstrained environments affected by long propagation delays. There are some important lessons to be learnt in dealing with acoustic interference from recent research in underwater networks; even though there, the focus is mostly on longdistance, low data rate communications. Significant recent efforts have attempted to address some of the challenges of interference modeling at the MAC layer [17], [18]. For example, it was shown in [17] that for slotted transmission the packet collision probability can be reduced by adding a guard band to each time slot to limit the effect of the spatial uncertainty of interference. However, (i) these solutions mainly rely on signaling exchanges that still suffer from the lowspeed of sound, and might result in under-utilization of the channel and therefore in low throughput; moreover, (ii) they look at the problem from a MAC perspective, exclusively. Furthermore, (iii) no previous work has modeled the temporal uncertainty of interference, i.e., its time-varying nature, and its cumulative effect. Finally, (iv) previous work is largely based on the protocol interference model, i.e., a packet is lost whenever two transmissions overlap at a receiver; which is not the case with advanced transmission schemes. Ultimately, existing models fail to capture the statistical behavior of timevarying and spatially uncertain ultrasonic channels.

Main Contributions. To address these challenges, in this work we develop for the first time (and validate through testbed experiments) new statistical models of ultrasonic interference. The models capture the unique spatial and temporal variability of unaligned ultrasonic interference through a new approach (that we refer to as $M$-sampling method) in which interference is characterized through a vector of measurements taken at multiple instants of time at each receiver during a given interval (i.e., time slot) to capture its statistical behavior. The effects of temporal uncertainty (i.e., the random transmission of different nodes on time-varying ultrasonic channels) on the interference level at each measurement point is modeled using generalized Nakagami probability distribution functions.

Based on this modeling framework, we formulate an optimization problem where the objective is to maximize the throughput achievable by mutually interfering nodes that are able to control their transmission profile (i.e., the probability to transmit over a series of time slots and available channels) and data generation rate under energy budget constraints. Then, we design (and validate through simulations and testbed experiments) new probabilistic throughput-maximizing distributed cross-layer control strategies based on these newly developed stochastic models of ultrasonic interference. A core feature of the proposed algorithm is that decisions at each node are taken based only on the second-order moment of ultrasonic interference (and not on its instantaneous level). The actual level of interference cannot in fact be known at the transmitter without significant delay and overhead, while its variance varies slowly in time. We show that the proposed joint optimization leads to up to nine times higher throughput with respect to optimizing the transmission profile or rate control individually.

The rest of the paper is organized as follows. In Section III, we describe the propagation characteristics of ultrasonic signals in human tissues, and based on our experimental measurement results, we propose statistical models of ultrasonic smallscale fading and interference, respectively. In Section IV, we formulate the optimization problem and then propose a distributed solution algorithm in Section V. Performance of the distributed algorithm is then evaluated through both simulation and testbed results in Section VI, and finally conclusions are drawn in Section VII.

\section{Channel And Interference Modeling}

Ultrasonic waves originate from the propagation of mechanical vibrations of particles in an elastic medium at frequencies above the upper limit for human hearing, i.e., $20 \mathrm{kHz}$. Acoustic propagation through a medium is governed by the acoustic wave equation (Helmhotz equation), which describes pressure variation over the three dimensions, $\nabla^{2} P-\frac{1}{c^{2}} \frac{\partial^{2} P}{\partial t^{2}}=0$, where $P(x, y, z, t)$ represents the acoustic pressure scalar field in space and time, and $c$ is the propagation speed in the medium 


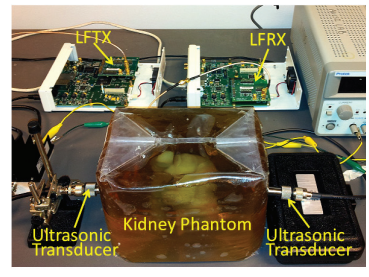

(a)

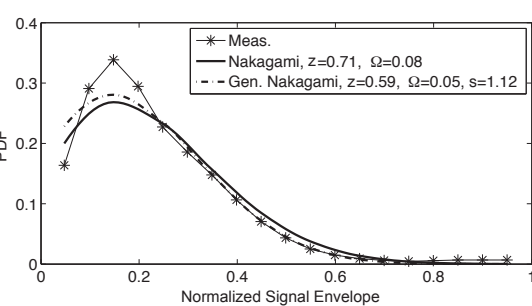

(b)
Fig. 1: (a) Ultrasonic experimental testbed; (b) Measurement and fitting results for the envelope fading of ultrasonic signals.

with a typical value of $1500 \mathrm{~m} / \mathrm{s}$ in blood and other soft tissues [4] (i.e., five orders of magnitude slower than RF propagation in air).

In [4] it was shown that attenuation can be significant and it increases (exponentially) with the distance between transmitter and receiver. Even with transmission distances of no more than a few tens of centimeters, due to the low speed of ultrasonic signals, the propagation delay can be rather large compared with the channel access period, leading to non-aligned interference and thus making time-division MAC protocols not efficient. Based on the experimental observations from our testbed measurements, next we propose a theoretically tractable interference model for ultrasonic propagation in human tissues. To this end, we first characterize the smallscale fading of the ultrasonic channel - for which unfortunately there is no literature available to date.

Channel Model. We conducted a series of experiments to measure the small-scale fading on the channel with the ultrasonic software defined testbed that we developed. The testbed consists of ultrasonic software defined nodes (uSDNs) communicating through physical medical phantoms (i.e., tissue-mimicking materials with acoustically accurate representations of anatomy) that emulate with high fidelity propagation through biological tissues. The uSDNs are implemented using the Universal Software Radio Peripheral (USRP) N210 software-defined radios interfaced with lowfrequency (LFRX and LFTX) daughterboards and ultrasonic transducers operating around $5 \mathrm{MHz}$ with a $-6 \mathrm{~dB}$ bandwidth of about $4 \mathrm{MHz}$. Phantoms are interposed between transmitters and receivers to emulate propagation through tissues. In Fig. 1(a) we show a sample experimental setup with a phantom mimicking propagation through a human kidney. We measured the faded envelope of ultrasonic signals, and the experimental result is least-square fitted with a Nakagami and a generalized Nakagami distribution. We found that the generalized Nakagami distribution fits the measurement results very well, as shown in Fig. 1(b). Results indicate that the ultrasonic signals are attenuated by both randomly-located and structured scatterers when propagating through the kidney, corresponding to a Nakagami parameter between 0.5 and 1 (0.59 with generalization parameter $s=1.12$ according to our measurements in the considered setting). To the best of our knowledge, these are the first measurements reported for ultrasonic communications in tissues.

Motivated by these experimental observations, we model the

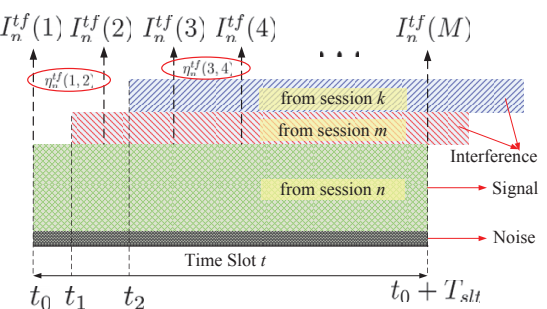

(a)

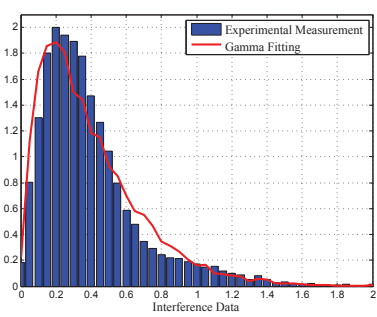

(b)
Fig. 2: (a) Interference Model; (b) Validation of the Gammadistribution-based interference model.

statistical characteristics of the channel fading coefficient $\rho_{t}$ for any time slot $t$ using a generalized Nakagami distribution function $\xi(x)$,

$$
\xi(x)=\mathrm{P}\left[\rho_{t}=x\right]=\frac{2 s z^{z} x^{2 s z-1}}{\Gamma(z) \Omega^{z}} e^{-\frac{z}{\Omega} x^{2 s}},
$$

where $z, \Omega$ and $s$ are the shaping, spreading, and generalization parameters of the generalized Nakagami distribution function that can be measured off-line or estimated online, and $\Gamma(z) \triangleq$ $\int_{0}^{\infty} x^{z-1} e^{-x} d x$ is the gamma function.

Then, the channel gain of the ultrasonic link in tissues from transmitter $m$ to receiver $n$ on sub-channel $f \in \mathcal{F}$ at time slot $t$, denoted by $h_{n m}^{t f}$, can be represented as

$$
h_{n m}^{t f}=H_{n m}^{f} \cdot\left(\rho_{t}\right)^{2} \text {, }
$$

where $H_{n m}^{f}$ represents the transmission attenuation that an ultrasonic signal transmitted on sub-channel $f \in \mathcal{F}$ experiences over a transmission distance $d_{n m}$ [4]. Denote $f_{c}$ as the central frequency of sub-channel $f$. Then, $H_{n m}^{f}$ can be represented as $H_{n m}^{f}=e^{-\beta\left(f_{c}\right) \cdot d_{n m}}$, where $\beta\left(f_{c}\right)$ (in $\left.\left[\mathrm{np} \cdot \mathrm{cm}^{-1}\right]\right)$ represents the amplitude attenuation coefficient that captures all the effects associated to energy dissipation from the ultrasonic beam. The parameter $\beta\left(f_{c}\right)$ can be further represented as $\beta=a \cdot\left(f_{c}\right)^{b}$, where $a$ (in $\left[\mathrm{np} \mathrm{m}{ }^{-1} \mathrm{MHz}^{-\mathrm{b}}\right]$ ) and $b$ are attenuation parameters characterizing the tissue that can be measured off-line. In the rest of the paper, we simplify $h_{n m}^{t f}, H_{n m}^{f}$ and $d_{n m}$ to $h_{n}^{t f}, H_{n}^{f}$ and $d_{n}$, respectively, for $n=m$.

Interference Model. The interference experienced at each receiver depends not only on the channel model described above, but also on the concurrent interfering but unsynchronized transmissions of other interfering nodes. Specifically, because of the non-negligible ultrasonic propagation delay, which may be much larger than the time duration of a time slot, signals transmitted simultaneously by different transmitters in general do not reach the receiver at the same time. This makes interference modeling for intra-body ultrasonic networks rather challenging.

To model the effect of non-aligned interference, we propose a new approach (that we refer to as $M$-sampling method) in which interference is characterized through a vector of measurements taken at multiple instants of time at each receiver during a given interval (i.e., time slot). Each receiver $n \in \mathcal{N}$ measures the received signal on each sub-channel $f \in \mathcal{F}$ in each time slot $t$ at a set $\mathcal{M}_{n}^{t f}$ with $\left|\mathcal{M}_{n}^{t f}\right|=M$ of time instants. An illustration is shown in Fig. 2(a).

Because of the non-aligned nature of interference, a signal 
from session $n \in \mathcal{N}$ that arrives at its intended receiver at the $l$-th measurement instant in time slot $t$, with $l \in \mathcal{M}_{n}^{t f}$ for any $f \in \mathcal{F}$, can receive interference from the transmission of any session $m \in \mathcal{N} / n$ occurring during time slot $\tilde{t}(m, n, t, l)$, which might be different from $t$. Denote the transmission probability corresponding to $\tilde{t}(m, n, t, l)$ as $\alpha_{m}^{\tilde{t}(m, n, t, l) f}$ (also $\alpha_{m}^{\tilde{t} f}(n, t, l)$ for notational convenience). Let $I_{n}^{t f}(l), l \in \mathcal{M}_{n}^{t f}$, denote the aggregate interference measured by the receiver node of session $n \in \mathcal{N}$ on sub-channel $f \in \mathcal{F}$ at the $l$ th measurement point in time slot $t$. Then, $I_{n}^{t f}(l)$ can be expressed as

$$
I_{n}^{t f}(l)=\sum_{m \in \mathcal{N} / n} P_{m}^{0} h_{n m}^{t f} \hat{\alpha}_{m}^{\tilde{t f}}(n, t, l), \forall l \in \mathcal{M}_{n}^{t f},
$$

where $\hat{\alpha}_{m}^{\tilde{f} f}(n, t, l)=1$ with probability $\alpha_{m}^{\tilde{t f}}(n, t, l)$ and 0 with probability $1-\alpha_{m}^{\tilde{t} f}(n, t, l)$.

Now we model the statistical characteristics of the interference level at each measurement time point $l$. With the previously discussed model of channel fading based on the generalized Nakagami distribution function, the probability density function of the aggregate interference at each measurement instant $I_{n}^{t f}(l)$ can be characterized by a Gamma distribution function

$$
\mathrm{P}\left[I_{n}^{t f}(l)=x\right]=\gamma_{n}^{t f l}(x)=x^{k_{n}^{t f l}} \frac{e^{-x / \theta_{n}^{t f l}}}{\Gamma\left(k_{n}^{t f l}\right)\left(\theta_{n}^{t f l}\right)_{n}^{t f l}},
$$

where $\Gamma\left(k_{n}^{t f l}\right)$ is the gamma function as in (1), while $k_{n}^{t f l}$ and $\theta_{n}^{t f l}$ are the shaping parameters of the Gamma distribution function depending on the transmission probabilities of all interferers $\hat{\alpha}_{m}^{\tilde{t} f}(n, t, l)$ in (3).

We validated the interference model through experiments, where we considered 10 interfering transmitters and one receiver randomly located around the kidney phantom. The power of each transmitter is set to $22 \mathrm{dBm}$, and the measurement period is set to $0.3 \mu \mathrm{s}$. We let each transmitter emit ultrasonic signals with probability 0.5 in each measurement period, and record the aggregate interference at the receiver. The probability density function of the measured interference is plotted in Fig. 2(b). Then, we fit the measured data using the Gamma distribution function with parameters $k_{n}^{t f l}$ and $\theta_{n}^{t f l}$ in (4) estimated as

$$
\begin{aligned}
& k_{n}^{t f l}=\left(\mu_{n}^{t f}(l)\right)^{2} /\left(\sigma_{n}^{t f}(l)\right)^{2}, \\
& \theta_{n}^{t f l}=\left(\sigma_{n}^{t f}(l)\right)^{2} / \mu_{n}^{t f}(l),
\end{aligned}
$$

with $\mu_{n}^{t f}(l)$ and $\sigma_{n}^{t f}(l)$ being the mean and standard deviation of the recorded interference levels at the considered measurement time point $l$. Clearly, a Gamma distribution fits the experimental measurements very well.

Modeling the Temporal Correlation. Since each transmission lasts for a time slot duration $T_{\text {slt }}$, measurements of aggregate interference at different time instants in each time slot can be closely correlated with each other. Let $\eta_{n}^{t f}(l, \tilde{l})$ with $l, \tilde{l} \in \mathcal{M}_{n}^{t f}$ represent the correlation coefficient between $I_{n}^{t f}(l)$ and $I_{n}^{t f}(\tilde{l})$. Then, $\eta_{n}^{t f}(l, \tilde{l})$ can be expressed as

$$
\eta_{n}^{t f}(l, \tilde{l})=\frac{\mathrm{E}\left[\left(I_{n}^{t f}(l)-\mu_{n}^{t f}(l)\right)\left(I_{n}^{t f}(\tilde{l})-\mu_{n}^{t f}(\tilde{l})\right)\right]}{\sigma_{n}^{t f}(l) \sigma_{n}^{t f}(\tilde{l})} .
$$

The interference measurements in $\mathcal{M}_{n}^{t f}$ can be grouped into $\left\{\tilde{\mathcal{M}}_{n}^{t f}(l)\right\}$ with each $\tilde{\mathcal{M}}_{n}^{t f}(l)$ consisting of a subset of measurements starting from the $l$-th. We adopt a thresholdbased grouping policy, which groups a number of consecutive interference measurements $l, l+1, \cdots, \tilde{l}$ into $\tilde{\mathcal{M}}_{n}^{t f}(l)$ so that the correlation coefficient between the interference levels at any two measurements in the group (which are not necessarily adjacent to each other) is greater than a threshold denoted as $\eta_{n}^{\text {th }}$, i.e., $\eta_{n}^{t f}\left(l_{1}, l_{2}\right) \geq \eta_{n}^{\text {th }}, l \leq l_{1}, l_{2} \leq \tilde{l}$. An example of the measurement grouping is shown in Fig. 2(a), where $I_{n}^{t f}(3)$ and $I_{n}^{t f}(4)$ are grouped together since $\eta_{n}^{t f}(3,4)>\eta_{n}^{\text {th }}$ while $I_{n}^{t f}(1)$ and $I_{n}^{t f}(2)$ are not.

\section{Problem Formulation}

Having developed the channel and interference models in the previous section, we now formulate and study a networklevel optimization problem for ultrasonic intra-body communications.

Network Description. Consider an ultrasonic intra-body area network with a set $\mathcal{N}$ of concurrent sessions with $|\mathcal{N}|=N$, each consisting of a transmitter-receiver pair. The available spectrum is divided into a set $\mathcal{F}$ of orthogonal sub-channels with $|\mathcal{F}|=F$ - the latter can be obtained on a code-division or frequency-division basis. Note that we consider multiple subchannels to keep the system model as general as possible. The transmission time is divided into consecutive time slots, which are further grouped into consecutive frames each consisting of a set $\mathcal{T}$ of time slots with $|\mathcal{T}|=T$.

Let $\boldsymbol{R}=\left(R_{n}\right)_{n \in \mathcal{N}}$ represent the data generation profile, i.e., the transport-layer data allowed in the network. Then, each source node $n \in \mathcal{N}$ introduces data in its queue at an average rate of $R_{n}$ [bit/s]. Because of the fast variability and high propagation delay of the ultrasonic channel, we assume that each session can obtain only statistical channel state information (CSI) at the transmitter side (i.e., no fast feedback is available). Therefore, unlike in traditional RF communications, each transmitter adopts a stochastic policy (based only on the estimated statistics of interference at the receiver, and not on its instantaneous value), to decide whether to transmit in a specific time slot and over which sub-channel to transmit. Let $\boldsymbol{\alpha}_{n}^{t}=\left(\alpha_{n}^{t f}\right)_{f \in \mathcal{F}}$ denote the data transmission vector of transmitter $n \in \mathcal{N}$ in time slot $t \in \mathcal{T}$, where $\alpha_{n}^{t f}$ represents the probability that session $n$ transmits a packet over sub-channel $f \in \mathcal{F}$. Then, the transmission profile of session $n \in \mathcal{N}$ in a time frame denoted as $\boldsymbol{\alpha}_{n}$ can be written as $\boldsymbol{\alpha}_{n}=\left(\boldsymbol{\alpha}_{n}^{t}\right)_{t \in \mathcal{T}}$. Let $\boldsymbol{\alpha}$ represent the transmission profile of all sessions in $\mathcal{N}$ and $\boldsymbol{\alpha}_{-n}$ represent the transmission policy vector of all sessions except $n$. Then, $\boldsymbol{\alpha}$ and $\boldsymbol{\alpha}_{-n}$ can be written as $\boldsymbol{\alpha}=\left(\boldsymbol{\alpha}_{n}\right)_{n \in \mathcal{N}}$ and $\boldsymbol{\alpha}_{-n}=\left(\boldsymbol{\alpha}_{m}\right)_{m \in \mathcal{N} / n}$, respectively. Note that this model includes deterministic policies (i.e., in which the probability to transmit over a specific time slot or channel is equal to one) as a special case.

Let $P_{n}^{0}$ denote the transmission power of user $n \in \mathcal{N},{ }^{1}$ $T_{\text {slt }}$ represent the time slot duration, and denote $E_{n}^{\max }$ as the

\footnotetext{
${ }^{1}$ We consider fixed transmission power since power control requires instantaneous CSI at the transmitter, which we assume to be unavailable.
} 
maximum energy available in each time frame consisting of $|\mathcal{T}|=T$ consecutive time slots. Then, we have

$$
\begin{aligned}
& \alpha_{n}^{t f} \geq 0, \forall n \in \mathcal{N}, \forall t \in \mathcal{T}, \forall f \in \mathcal{F} \\
& \alpha_{n}^{t f} \leq 1, \forall n \in \mathcal{N}, \forall t \in \mathcal{T}, \forall f \in \mathcal{F} \\
& \sum_{f \in \mathcal{F}} \alpha_{n}^{t f} \leq 1, \forall n \in \mathcal{N}, \forall t \in \mathcal{T} \\
& \sum_{t \in \mathcal{T}}\left(R_{n} E_{n}^{0}+\sum_{f \in \mathcal{F}} \alpha_{n}^{t f} P_{n}^{0}\right) T_{\text {slt }} \leq E_{n}^{\max }, \forall n \in \mathcal{N}
\end{aligned}
$$

where $E_{n}^{0}$ represents the energy [J] consumed by source node $n$ to generate and process (e.g., A/D conversion, source encoding) one bit of data. Constraint (10) imposes that each session transmit on at most one sub-channel in each time slot; Constraint (11) imposes a balance between the energy needed for (i) data processing and generation and (ii) transmission for throughput maximization, under a given energy budget and limiting radiation to specific safety levels [4], [11]. Note that it is essential to incorporate rate control into the optimization framework, since in some scenarios of interest it might be very difficult to determine the optimal rate in advance. Consider for example a dynamic intra-body network with a set of miniaturized sensors cruising along the blood vessels to conduct multi-site measurement of physiological quantities of interest. As discussed in further detail in Section VI, without rate control, trivially allocating too high a portion of the energy budget to data generation leads to growing queueing delay that could result in high packet drop rates (therefore reducing the throughput). The underlying queueing model will be introduced later in this section.

Let $U_{n}\left(R_{n}, \boldsymbol{\alpha}_{n}, \boldsymbol{\alpha}_{-n}\right)$ represent the throughput of session $n \in \mathcal{N}$, which depends on its data generation rate $R_{n}$, transmission profile $\boldsymbol{\alpha}_{n}$ and also on the transmission profiles of all interfering sessions in $\mathcal{N} / n$. Then, with the energy constraint in (11), the objective of each transmitter $n \in \mathcal{N}$ is to maximize its throughput $U_{n}\left(R_{n}, \boldsymbol{\alpha}_{n}, \boldsymbol{\alpha}_{-n}\right)$ by jointly adjusting its data generation rate $R_{n}$ and the transmission profile $\boldsymbol{\alpha}_{n}$, based on the statistical behavior of the observed interference caused by other interfering transmitters. To formalize the optimization problem, next we derive the mathematical expression for throughput $U_{n}\left(R_{n}, \boldsymbol{\alpha}_{n}, \boldsymbol{\alpha}_{-n}\right)$.

Throughput Derivation. Due to the non-aligned interference as shown in Fig. 2(a), the experienced signal-to-interferenceplus-noise ratio (SINR) in a time slot does not remain constant even if the channel is assumed to be slow-fading. This results in a fast-fading channel in each time slot. ${ }^{2}$ If the measurement instants in each time slot are uniformly spaced, ${ }^{3}$ then the outage probability of each session $n \in \mathcal{N}$ in time slot $t$ over sub-channel $f \in \mathcal{F}$, denoted as $O_{n}^{t f}\left(\boldsymbol{\alpha}_{-n}\right)$, can be expressed as [19]

$$
O_{n}^{t f}\left(\boldsymbol{\alpha}_{-n}\right) \triangleq \mathrm{P}\left[\sum_{l \in \mathcal{L}\left(\mathcal{M}_{n}^{t f}\right)} \frac{\left|\tilde{\mathcal{M}}_{n}^{t f}(l)\right|}{\left|\mathcal{M}_{n}^{t f}\right|} C(n, t, f, l)<R_{n}^{0}\right]
$$

\footnotetext{
${ }^{2}$ Different from fast fading in in-air radio-frequency communications, which is caused by the channel variation itself [19], here fast fading is caused by the non-aligned interference.

${ }^{3}$ Different measurement distributions will be studied in our future work, e.g., random distribution, compressive-sampling-based measurements.
}

where $\mathcal{L}\left(\mathcal{M}_{n}^{t f}\right)$ represents the set of beginning measurements of each group in $\left\{\tilde{\mathcal{M}}_{n}^{t f}\right\}$ defined in Section III, and

$$
C(n, t, f, l)=B \log _{2}\left(1+P_{n}^{0} h_{n}^{t f} /\left[\left(\delta_{n}^{f}\right)^{2}+I_{n}^{t f}(l)\right]\right)
$$

represents the achievable capacity during measurement group $\tilde{\mathcal{M}}_{n}^{t f}(l)$ with $\left(\delta_{n}^{f}\right)^{2}$ being the ambient noise power at the receiver node of session $n \in \mathcal{N}$ over sub-channel $f \in \mathcal{N}$; $B$ represents the bandwidth $[\mathrm{Hz}]$ of each sub-channel, and $R_{n}^{0}$ is the target rate required to transmit a packet in a time slot; $I_{n}^{t f}(l)$ is the aggregate interference given in (3).

Then, the average capacity of session $n \in \mathcal{N}$, denoted as $C_{n}\left(\boldsymbol{\alpha}_{n}, \boldsymbol{\alpha}_{-n}\right)$, can be expressed as

$$
C_{n}\left(\boldsymbol{\alpha}_{n}, \boldsymbol{\alpha}_{-n}\right)=\frac{1}{|\mathcal{T}|} \sum_{t \in \mathcal{T}} C_{n}^{t}\left(\boldsymbol{\alpha}_{n}, \boldsymbol{\alpha}_{-n}\right),
$$

with

$$
C_{n}^{t}\left(\boldsymbol{\alpha}_{n}, \boldsymbol{\alpha}_{-n}\right)=\sum_{f \in \mathcal{F}} \alpha_{n}^{t f}\left(1-O_{n}^{t f}\left(\boldsymbol{\alpha}_{-n}\right)\right) R_{n}^{0} .
$$

Finally, if we assume as in [20] that packet arrivals of each user $n \in \mathcal{N}$ follow a Poisson process with average arrival rate $R_{n}$ [bit/s] and that the service time of each packet with length $L_{n}$ bits follows an exponential distribution, the queue of each user $n \in \mathcal{N}$ can be modeled as an $M / M / 1$ queue [21]. Let $P_{n}^{\text {dly }}\left(R_{n}, \boldsymbol{\alpha}_{n}, \boldsymbol{\alpha}_{-n}\right)$ represent the packet loss rate of user $n$ caused by exceeding the maximum queueing delay $T_{n}^{\text {th }}$. Then, $P_{n}^{\text {dly }}\left(R_{n}, \boldsymbol{\alpha}_{n}, \boldsymbol{\alpha}_{-n}\right)$ can be expressed as

$$
P_{n}^{\text {dly }}\left(R_{n}, \boldsymbol{\alpha}_{n}, \boldsymbol{\alpha}_{-n}\right)=e^{-\left(C_{n}\left(\boldsymbol{\alpha}_{n}, \boldsymbol{\alpha}_{-n}\right)-R_{n}\right) \frac{T_{n}^{\text {th }}}{L_{n}}},
$$

and the throughput of each session $n \in \mathcal{N}$, denoted as $U_{n}\left(R_{n}, \boldsymbol{\alpha}_{n}, \boldsymbol{\alpha}_{-n}\right)$, can be expressed as

$$
U_{n}\left(R_{n}, \boldsymbol{\alpha}_{n}, \boldsymbol{\alpha}_{-n}\right)=R_{n}\left(1-P_{n}^{\mathrm{dly}}\left(R_{n}, \boldsymbol{\alpha}\right)-P_{n}^{\mathrm{err}}\right),
$$

where $P_{n}^{\text {err }}$ represents the residual packet error rate due to non-perfect channel coding/decoding techniques, which is considered fixed in this work.

Problem Statement. So far, we have derived an expression for the throughput of each session. Then, our objective is design lightweight, asynchronous and distributed resource allocation strategies to maximize the achievable throughput $U_{n}\left(R_{n}, \boldsymbol{\alpha}_{n}, \boldsymbol{\alpha}_{-n}\right)$ defined in (17) for each session $n \in \mathcal{N}$, with given energy budget $E_{n}^{\max }$ while keeping the radiated energy level within safety limits, by jointly controlling the stochastic channel access profile $\boldsymbol{\alpha}_{n}$ and regulating the data generation rate $R_{n}$.

To this end, next we present the proposed distributed algorithm, which is referred to as D-ROSA (Distributed Rate cOntrol and Stochastic channel Access), and then discuss several issues related to practical implementation of the algorithm and convergence of the algorithm. To provide a performance benchmark, we also design a globally optimal but centralized solution algorithm, which will be briefly discussed in Section VI. 


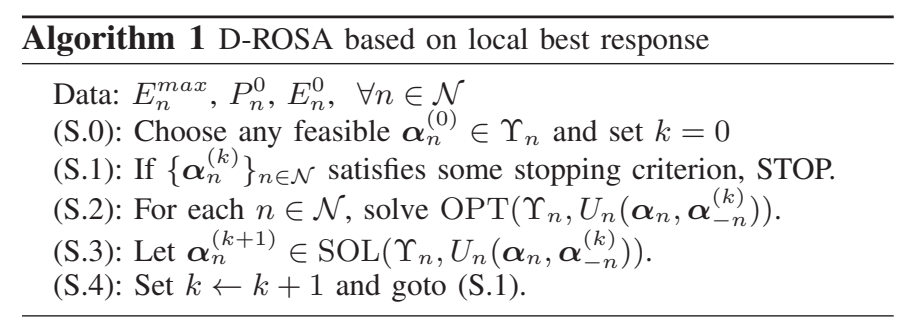

\section{Distributed Solution Algorithm}

The D-ROSA algorithm is based on a local best-response strategy. Each transmitter iteratively solves the problem of joint rate control and transmission probability profile adaption based on a local observation of the second order statistics of the aggregate interference at the receiver.

Algorithm. We let $\Upsilon_{n}=\left\{\left(R_{n}, \boldsymbol{\alpha}_{n}\right)\right\}$ represent the domain set of session $n \in \mathcal{N}$, which consists of all possible combinations of $R_{n}$ and $\boldsymbol{\alpha}_{n}$, as defined in (8)-(11). Then, for a given (fixed) transmission profile of all other sessions in $\mathcal{N} / n$, the individual optimization problem of session $n \in \mathcal{N}$ denoted as $\operatorname{OPT}\left(\Upsilon_{n}, U_{n}\right)$ with $U_{n}=U_{n}\left(R_{n}, \boldsymbol{\alpha}_{n}, \boldsymbol{\alpha}_{-n}\right)$, can be represented as

$$
\begin{array}{cl}
\text { Given : } & E_{n}^{\max }, E_{n}^{0}, P_{n}^{0}, \boldsymbol{\alpha}_{-n} \\
\underset{R_{n}, \boldsymbol{\alpha}_{n}}{\operatorname{maximize}:} & U_{n}\left(R_{n}, \boldsymbol{\alpha}_{n}, \boldsymbol{\alpha}_{-n}\right) \\
\text { subject to : } & \text { Transmission constraints : (8) }-(10) \\
& \text { Energy constraint : (11) }
\end{array}
$$

Then, the distributed best-response-based algorithm can be formalized as in Algorithm 1, where $\operatorname{SOL}\left(\Upsilon_{n}, U_{n}\right)$ represents the solution set of $\operatorname{OPT}\left(\Upsilon_{n}, U_{n}\right)$.

Note that Algorithm 1 is a Jacobi version of the distributed algorithm [14]. In practice, different sessions do not need to be synchronized when updating their own transmission probability profile, which results in a Gauss-Seidel-like implementation of the algorithm [14]. Note that at each iteration in Algorithm 1, each session solves an individual optimization problem formulated in (18), where the transmission probability profile of all other sessions $\boldsymbol{\alpha}_{-n}$ is assumed to be known as input, which is not the case in practice. Since we focus on fully distributed algorithms without any message exchange among different sessions, then a natural question that arises is: how can each session adjust its transmission probability profile based on the profile of the other sessions?

Proposition 1. It is sufficient for each session $n \in \mathcal{N}$ to estimate the statistical effects of aggregate interference from all other sessions in $\mathcal{N} / n$ with transmission profile $\boldsymbol{\alpha}_{-n}$ on its own throughput by recording the first-and second-order moments of the interference level observed at its intended receiver.

Proof. Recall that in Section III, we verified that the aggregate interference follows a Gamma distribution. Then, according to (5) and (6), the probability density function of interference can be exactly determined by (first-order) mean and (second-order) variance.
Each session $n \in \mathcal{N}$ periodically transmits an updated estimate of the interference mean and variance back to its transmitter. Then, based on (3)-(15), the transmitter can calculate the outage probability profile $\left(O_{n}^{t f}\right)_{t \in \mathcal{T}, f \in \mathcal{F}}$ and adjust its data generation rate $R_{n}$ and transmission profile $\boldsymbol{\alpha}_{n}$ to maximize its own individual throughput $U_{n}\left(R_{n}, \boldsymbol{\alpha}_{n}, \boldsymbol{\alpha}_{-n}\right)$ as given in (16) and (17). Since the expression in (17) is still nonconcave, each optimization problem $\operatorname{OPT}\left(\Upsilon_{n}, U_{n}\right)$ is nonconvex. In Theorem 1, we show however that the globally optimal solution of each $\operatorname{OPT}\left(\Upsilon_{n}, U_{n}\right)$ can still be obtained with polynomial-time algorithms.

Theorem 1. Each individual optimization problem in $\operatorname{OPT}\left(\Upsilon_{n}, U_{n}\right)$ can be solved by solving an equivalent convex optimization problem.

Proof. It can be proven that the objective function $U_{n}\left(R_{n}, \boldsymbol{\alpha}_{n}, \boldsymbol{\alpha}_{-n}\right)$, with given $\boldsymbol{\alpha}_{-n}$, is a log-concave function. Moreover, all constraints in (8)-(11) are linear constraints, hence the resulting domain set is convex (also bounded and closed). Therefore, maximizing $U_{n}\left(R_{n}, \boldsymbol{\alpha}_{n}, \boldsymbol{\alpha}_{-n}\right)$ is equivalent to maximizing its logarithm, which is a convex optimization problem, whose globally optimal solution can be solved in polynomial computational complexity using standard convex optimization techniques $[22, \S 11]$. Proof of the log-concavity of $U_{n}$ follows the rule that affine mapping preserves convexity of function [22, P79, §3.2.2].

Convergence Analysis. We now provide some results on the convergence property of the proposed algorithm.

Theorem 2. Given the number of available channels $|\mathcal{F}|$ and the number of concurrent sessions $|\mathcal{N}|$, Algorithm 1 converges to a stationary point of the problem of joint rate control and stochastic channel access, if the number of jointly optimized time slots, i.e., $|\tau|$, is sufficiently large. At this point, for each session there is no incentive to unilaterally deviate from its current transmission strategy.

Proof. The theorem can be proven by showing that the individual utility function $U_{n}\left(R_{n}, \boldsymbol{\alpha}_{n}, \boldsymbol{\alpha}_{-n}\right)$ in (18) satisfies the condition in [24, Proposition 3]. Details are omitted because of space limitations.

Intuitively, with a large number of jointly optimized time slots, changing the transmission probability in a time slot for a session only affects the overall achievable capacity of the considered session slightly. Additionally, due to the linearity of (15) with respect to $\alpha_{n}^{t f}$, each session assigns nonzero transmission probability to only a subset of the jointly optimized time slots. The larger $|\tau|$ is, the more likely it is that the time slot subsets for different sessions are disjoint with each other, which implies convergence of the algorithm. In practice, Algorithm 1 converges very fast to a stable zone with only limited $|\tau|$, as shown in Section VI.

Practical Considerations. Note that Algorithm 1 does not require each receiver to send interference parameters (i.e., mean and variance) back to its transmitter at every time slot. Since the algorithm is designed based on a statistical characterization of the interference, feedback needs to be invoked only when there is a noticeable change in the statistical 


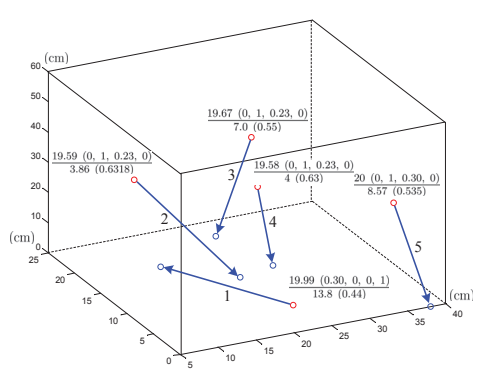

(a)

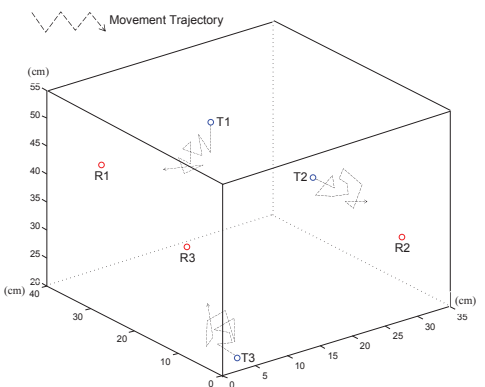

(b)

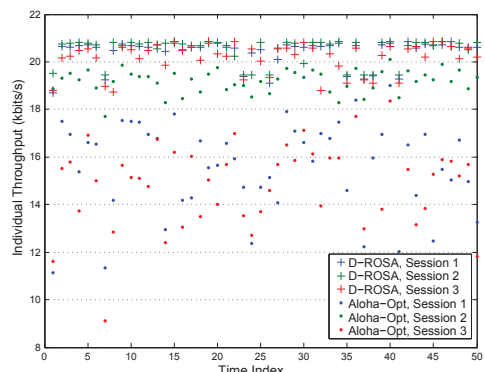

(c)

Fig. 3: (a) Individual throughput and transmission probability profile with 5-sessions; (b) The transmitter of each session moves following the Random waypoint model [23]; (c) Individual throughput by D-ROSA and Aloha-Opt in scenario (b).

TABLE I: Simulation Parameters

\begin{tabular}{|c|c|c|}
\hline Para. & Physical Meaning & Value \\
\hline \hline & Communication area & $40 \times 40 \times 60\left(\mathrm{~cm}^{3}\right)$ \\
$\beta$ & Number of nodes & $4,8,6,10,20,50$ \\
& Amplitude attenuation & 0.1 (ultrasonic \\
coefficient & propagation in blood) \\
$F=|\mathcal{F}|$ & Number of sub-channels & 1,2 \\
$T=|\mathcal{T}|$ & Number of time slots & $1,3,4,5,7,9,11$ \\
& in a frame & $2,4,6,8,10,30,50$, \\
$R_{n}$ & Data generation rate & $80,110(\mathrm{kbit} / \mathrm{s})$ \\
& Sub-channel Bandwidth & $50(\mathrm{kHz})$ \\
$L_{n}$ & Packet length & $100(\mathrm{bit})$ \\
$T_{\text {slt }}$ & Time slot duration & $0.5 \times 10^{-3}(\mathrm{~s})$ \\
\hline
\end{tabular}

characteristics of interference compared with the last feedback, typically after tens or hundreds of time slots. Moreover, in each feedback message only mean and variance of the inference are transmitted. Furthermore, as discussed above, each transmitter only needs to solve a convex optimization problem with polynomial complexity with respect to the number of jointly optimized time slots upon receiving feedback. Therefore, the resulting communication and computational complexity is in practice low.

It is worth pointing out that since feedback information (including packet acknowledgement information) is short, it can be sent back to the transmitter based on reliable transmission schemes, e.g., using repetition coding [25], or CDMA with high spreading gain or other reliable coding techniques.

\section{Vi. Performance Evaluation}

Simulation Setup. Some key simulation parameters are summarized in Table I for the reader's convenience. For performance comparison, we implemented Aloha and its variations. To the best of our knowledge, there are no other existing schemes designed for ultrasonic networking in intrabody environments. Moreover, we do not compare against CSMA/CA-based schemes because carrier sensing is known to be ineffective with high propagation delays [26]. Specifically, we implemented three distributed schemes: (i) Aloha with persistence probability individually and locally optimized for each session in each time slot (Aloha-Opt), (ii) D-ROSA without considering rate control (WoRC), and iii) Aloha-Opt without considering rate control (Aloha-WoRC). To provide an upper-bound performance benchmark for D-ROSA, we also designed and implemented a centralized but globally optimal solution algorithm (referred to as C-ROSA). Given the nonconvexity of the centralized optimization problem, we designed the algorithm based on a combination of branch-and-bound framework and reformulation linearization technique (RLT). The algorithm searches for the globally optimal solution of the centralized version of the optimization problem formulated in (18) by iteratively updating global upper and lower bounds on the social objective function (i.e., sum throughput) until some stopping criterion is satisfied. In our experiment, we consider the algorithm to have converged when the global lower bound is greater than $95 \%$ of the global upper bound.

Case Study. First, as a case study we show the effect of multi-slot joint optimization on the individual throughput performance for a five-session intra-body network, as shown in Fig. 3(a). We observe that significant throughput gains can be obtained by D-ROSA compared with single-slot-based channel access (Aloha-Opt). For example, a $5 \times$ throughput gain is obtained by session 2 . For session 2 , a throughput of $19.59 \mathrm{kbit} / \mathrm{s}$ is achieved by transmitting with probability 1 and 0.23 in the second and third time slot in each frame, while keeping silent in the first and fourth (as indicated in the numerator in the figure). In the single-slot optimization, the session chooses to access the channel with probability 0.6318 in every time slot, and as a result, a throughput of only $3.86 \mathrm{kbit} / \mathrm{s}$ can be achieved. By averaging over the five sessions, a $3.3 \times$ throughput is achieved by D-ROSA. Indeed, we found that avoiding interference by adding a guard band to each time slot as proposed in [17] for underwater acoustic networks can be a natural outcome of optimizing the transmission strategy based on our framework. However, our framework leads to fully distributed solutions, while the approach in [17] needs global network information, and hence is not suited for fast time-varying networks.

Additional examples are presented in Fig. 3(b) and (c) by considering mobility of the transmitters. We consider three interfering sessions, and let each transmitter move randomly within a certain area (e.g., the targeted area of spatial-, temporal- and dosage-controlled medical applications [10]) following the Random waypoint model [23], as shown by the movement trajectory in Fig. 3(b). From Fig. 3(c) we can see that, D-ROSA considerably outperforms Aloha-Opt (i.e., Aloha with individually optimized persistence probability) in 


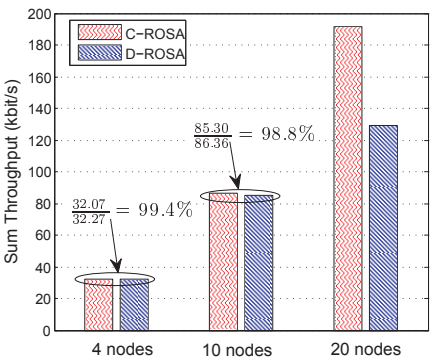

(a)

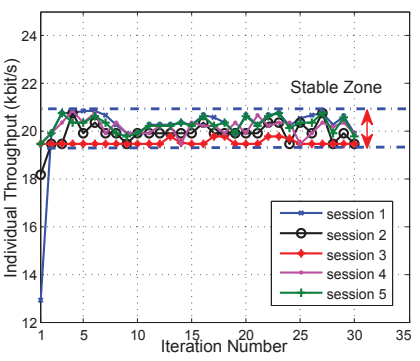

(b)
Fig. 4: (a) Throughput comparison between D-ROSA and C-ROSA; (b) The distributed algorithm converges to a stable zone very quickly.

terms of individual throughput in all tested network topologies and for all the three sessions.

Optimality and Convergence. Optimality and convergence properties of D-ROSA are studied in Fig. 4(a) and (b), respectively. From Fig. 4(a) we see that D-ROSA achieves nearly-optimal sum throughput in intra-body networks with a moderate number of nodes. For example, in 10-node intrabody networks $98.8 \%$ of the global optimum can be achieved by D-ROSA. Results also indicate a moderate performance gap between D-ROSA and C-ROSA when the number of nodes is large, e.g., around $70 \%$ of the optimum can be achieved. Note that this is achieved in a distributed way and with no message exchange. Cooperative strategies, requiring however message exchange among different sessions, were shown in our previous work to be able to partially fill this gap. However, for low densities of nodes that are envisioned in most practical applications, lightweight strategies with minimal message exchange like D-ROSA seem to be more appealing. From Fig. 4(b), we see that D-ROSA can quickly converge to a stable zone (always within several iterations in the tested instances).

The effect of multi-slot optimization on sum throughput is further studied in Fig. 5(a), with $T$ varying from 1 to 11 in steps of 2 . By comparing the case $T=3$ with $T=1$, we observe that D-ROSA always obtains throughput gains. Also, in an intra-body network with a moderate number of nodes, e.g., $4-16$ in Fig. 5(a), jointly optimizing more than three time slots can only slightly increase the sum throughput. This is because in a network with little or moderate interference, a lower number of time slots is sufficient to provide the degrees of freedom needed by the involved sessions to avoid creating excessive interference to one another. An extreme case is the single-session network without interference, where there is no need for the session to perform multi-slot joint optimization. The benefit is more obvious in a high-interference network, e.g., in a 20-node intra-body network, where a maximum $9.4 \times$ throughput gain can be achieved by jointly optimizing over 11 time slots.

The impact of rate control on the sum throughput is studied in Fig. 5(b), with 4, 8 and 16 nodes, and data generation rate $R_{n}$ fixed to different values from 0 to $110 \mathrm{kbit} / \mathrm{s}$ (for the algorithm WoRC). Compared with D-ROSA, the sum throughput of WoRC degrades considerably once the data generation rate $R_{n}$ is fixed to a given value for each session. While smaller values of $R_{n}$ directly degrade the sum throughput, injecting too much data into the network leads to a more congested queue and also less energy available for data transmission with the total energy constraint in (11). Note that, results in Fig. 5(b) are averaged over 100 simulations by varying the network topology. For a given specific intra-body network, if the rate $R_{n}$ can be carefully tailored, e.g., fixed to the optimum, then the resulting throughput of WoRC will coincide with D-ROSA. Doing so however is less flexible in practice for dynamic networks. For example, to measure in real-time specific blood components (e.g., the level of glucose) or to monitor certain tissues for micro-range and even multi-view imaging, a set of implanted sensors may need to move around as in Fig. 3(b), or cruise within a certain body area along blood vessels to conduct multi-point measurements. In this case, it would be very difficult to determine the optimal sampling rate in advance.

In Fig. 5(c), we compare the sum throughput achieved by the four distributed algorithms (D-ROSA, Aloha-Opt, WoRC, and Aloha-WoRC) in a 50-node network. While, as expected, D-ROSA outperforms all the others, it is somewhat surprising that the throughput achieved by Aloha-Opt can be much lower than that of Aloha-WoRC. This implies that, in a highinterference network, rate control must be jointly applied with multi-slot optimization, or otherwise each session will inject large amounts of data into the network to optimize its own individual throughput, while the other sessions have no way to avoid the resulting high interference by Aloha-Opt based on which each session optimizes its transmission probability by considering single time slot only.

Testbed Validation. We have also validated the effectiveness of the proposed algorithm by implementing it on the ultrasonic software-defined testbed discussed in Section III. We first briefly introduce the experimental setup. The experiment consists of two ultrasonic nodes that communicate through a human-kidney phantom. Two ultrasonic transducers are located on opposite sides of the phantom at a distance of $10 \mathrm{~cm}$. Time is divided in slots of $100 \mathrm{~ms}$ each, and each session can transmit at most one packet of 96 bytes per time slot. At the physical layer, we have implemented an orthogonal frequency-division multiplexing (OFDM) transmission scheme. We set the number of total subcarriers to 64 , of which 48 are actually used for data transmission, over a bandwidth of approximately $200 \mathrm{kHz}$ centered around $5 \mathrm{MHz}$, i.e., the central frequency of the ultrasonic transducer in use. The cyclic prefix is set to 16 samples. Each subcarrier is BPSK-modulated. This results in a physical layer data rate of approximately $120 \mathrm{kbit} / \mathrm{s}$.

To guarantee repeatability of the experiments, we generate interference from co-located transceivers by artificially injecting interference at the transmitter, and multiplying each session for a stochastic component that follows a Nakagami distribution, as discussed in Section III. We consider a maximum of five concurrent interfering sessions. In each time slot, each session transmits with a probability $p \in\{0.35,0.5,0.65,0.8\}$.

In Fig. 5(d), we plot the packet drop rate against the number of jointly optimized time slots. We consider two scenarios with 


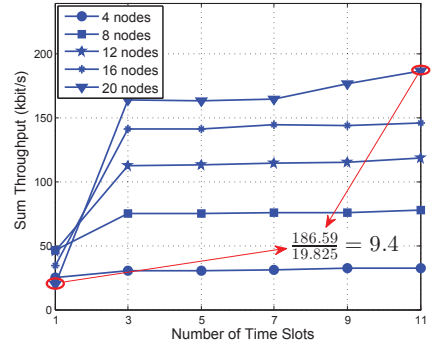

(a)

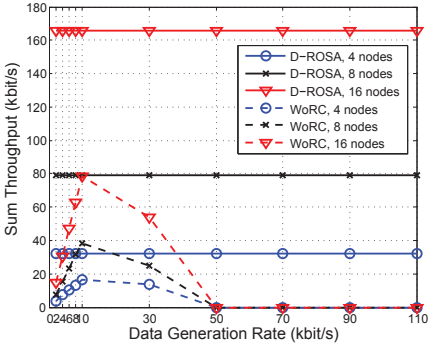

(b)

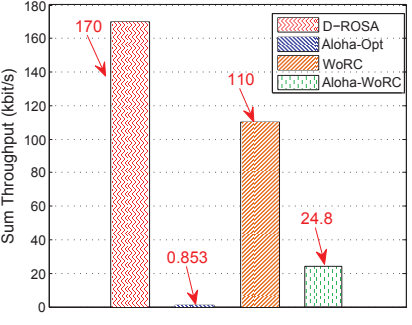

(c)

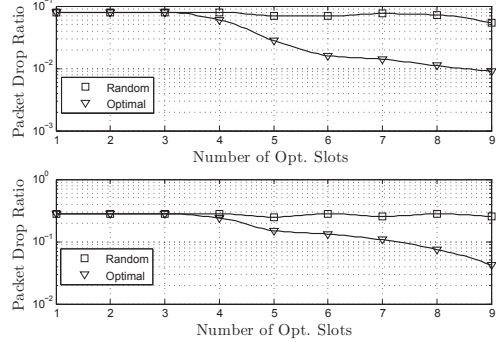

(d)

Fig. 5: (a) Sum throughput achieved with different number of time slots in a frame and number of nodes; (b) Sum throughput against data generation rate with different number of nodes; (c) Sum Throughput of different algorithms in a 50-node network; (d) Testbed validation: packet drop rate against the number of jointly optimized time slots. different interference levels, i.e., $S I N R=13 \mathrm{~dB}$ in Fig. $5 \mathrm{~d}$ (top) and $S I N R=10 \mathrm{~dB}$ in Fig. 5d (bottom). For comparison, we compare D-ROSA with a random channel access, where the transmitting node selects randomly three time slots in a time frame to transmit. We observe that the resulting packet drop ratio consistently decreases as more time slots are jointly considered. When jointly considering 9 time slots, up to 10 times lower packet drop rate can be achieved compared to random channel access in the case of $S I N R=13 \mathrm{~dB}$, while 6 times lower than for $S I N R=10 \mathrm{~dB}$.

\section{CONCLUSIONS}

We investigated for the first time algorithms for cross-layer control of functionalities in ultrasonic intra-body area networks. We focused on the design of lightweight, asynchronous, and distributed algorithms for joint stochastic channel access and rate control optimization, with the objective of maximizing the network throughput for a given energy budget.

We first developed a statistical model of the spatial and temporal variability of ultrasonic interference. Then, we formulated a throughput maximization problem under energy budget constraints and proposed a distributed solution algorithm. Extensive simulation results showed considerable throughput gains compared with Aloha-based channel access, and that separate rate control could lead to rather poor performance in intra-body environments. Testbed results have also been presented to validate effectiveness of the algorithm.

\section{REFERENCES}

[1] "Medtronic Pain Neurostimulation System." [Online]. Available: http://www.medtronic.com/

[2] “The CardioMEMS HF System.” [Online]. Available: http://www.sjm. com/cardiomems/

[3] "Microsemi, Implantable Medical Transceivers." [Online]. Available: http://www.microsemi.com/

[4] G. E. Santagati, T. Melodia, L. Galluccio, and S. Palazzo, "Ultrasonic Networking for e-Health Applications," IEEE Wireless Communications Magazine, vol. 20, no. 4, pp. 74-81, Aug. 2013.

[5] _ - "UsWB: Medium Access Control and Rate Adaptation for Ultrasonically Networked Implantable Sensors," IEEE/ACM Transactions on Networking, 2015.

[6] G. E. Santagati and T. Melodia, "Sonar Inside Your Body: Prototyping Ultrasonic Intra-body Sensor Networks," in Proc. of IEEE Conference on Computer Communications (INFOCOM), Toronto, Canada, April 2014.

[7] G. Oberholzer, P. Sommer, and R. Wattenhofer, "SpiderBat: Augmenting Wireless Sensor Networks With Distance and Angle Information," in Proc. of IEEE/ACM Intl. Conf. on Information Processing in Sensor Networks (IPSN), Chicago, IL, April 2011.

[8] F.L. Thurstone, H.E. Melton, "Biomedical Ultrasonics," IEEE Trans. on Ind. Electr. and Contr. Instrum., vol. 17, no. 2, pp. 167-172, April 1970.

[9] R. Smith, A. Arca, X. Chen, L. Marques, M. Clark, J. Aylott, and M. Somekh, "Design and Fabrication of Ultrasonic Transducers with Nanoscale Dimensions," Journal of Physics, vol. 278, no. 1, pp. 1-4, Jan. 2011.

[10] S. Mura, J. Nicolas, and P. Couvreur, "Stimuli-responsive Nanocarriers for Drug Delivery," Nature Materials, vol. 12, pp. 991-1003, Nov. 2013.

[11] T. Hogg and R. A. Freitas, "Acoustic Communication for Medical Nanorobots," Nano Communication Networks (Elsevier), vol. 3, no. 2, pp. 83-102, Feb. 2012.

[12] A. Y. Cheung and A. Neyzari, "Deep Local Hyperthermia for Cancer Therapy: External Electromagnetic and Ultrasound Techniques," Cancer Res. (Suppl.), vol. 44, no. 9, pp. 4736-4744, Oct. 1984.

[13] A. Kiourti, K. A. Psathas, and K. S. Nikita, "Implantable and Ingestible Medical Devices With Wireless Telemetry Functionalities: A Review of Current Status and Challenges," Bioelectromagnetics (Wiley), vol. 35, no. 1, pp. 1-15, Jan. 2014.

[14] D. P. Bertsekas and J. N. Tsitsiklis, Parallel and Distributed Computation: Numerical Methods. Upper Saddle River, NJ, USA: Prentice-Hall, Inc., 1989.

[15] Y. T. Hou, Y. Shi, and H. D. Sherali, "Optimal Spectrum Sharing for Multi-hop Software Defined Radio Networks," in Proc. of IEEE Intl. Conf. on Computer Communications (INFOCOM), Anchorage, Alaska, May 2007.

[16] X. Fang, D. Yang, and G. Xue, "Resource Allocation in Loadconstrained Multihop Wireless Networks," in Proc. of IEEE Intl. Conf. on Computer Communications (INFOCOM), Orlando, Fl, March 2012.

[17] A. Syed, W. Ye, J. Heidemann, and B. Krishnamachari, "Understanding Spatio-Temporal Uncertainty in Medium Access with ALOHA Protocols," in Proc. of ACM International Workshop on UnderWater Networks (WUWNet), Montreal, Quebec, Canada, Sep. 2007.

[18] Z. Zhou, Z. Peng, J.-H. Cui, and Z. Jiang, "Handling Triple Hidden Terminal Problems for Multichannel MAC in Long-Delay Underwater Sensor Networks," IEEE Transactions on Mobile Computing, vol. 11, no. 1, pp. 139-154, Jan. 2012.

[19] D. Tse and P. Viswanat, Fundamentals of Wireless Communication. USA: Cambridge University Press, 2005.

[20] X. Zhu, E. Setton, and B. Girod, "Congestion-Distortion Optimized Video Transmission Over Ad Hoc Networks," EURASIP Signal Processing: Image Communication, vol. 20, no. 8, pp. 773-783, Sept. 2005.

[21] D. Bertsekas and R. Gallager, Data Networks. USA: Prentice Hall, 2000.

[22] S. Boyd and L. Vandenberghe, Convex Optimization. Cambridge University Press, 2004.

[23] T. Camp, J. Boleng, and V. Davies, "A Survey of Mobility Models for Ad Hoc Network Research," Wireless Communications and Mobile Computing, vol. 2, no. 5, pp. 483-502, Aug. 2002.

[24] G. Scutari, D. P. Palomar, F. Facchinei, and J.-S. Pang, "Monotone Games for Cognitive Radio Systems," in Distributed Decision-Making and Control. Eds. Anders Rantzer and Rolf Johansson, Lecture Notes in Control and Information Sciences Series, Springer Verlag, 2011.

[25] A. A. Ali and I. A. Al-Kadi, "On the Use of Repetition Coding with Binary Digital Modulations on Mobile Channels," IEEE Transactions on Vehicular Technology, vol. 38, no. 1, pp. 14-18, Feb. 1989.

[26] D. Pompili, T. Melodia, and I. F. Akyildiz, "A CDMA-Based Medium Access Control for Underwater Acoustic Sensor Networks," IEEE Transactions on Wireless Communications, vol. 8, no. 4, pp. 1899-1909, April 2009. 